\title{
Near-infrared spectroscopy of the Sun and HD 20010
}

\section{Compiling a new line list in the near-infrared ${ }^{\star}$}

\author{
D. T. Andreasen ${ }^{1,2}$, S. G. Sousa ${ }^{1}$, E. Delgado Mena ${ }^{1}$, N. C. Santos ${ }^{1,2}$, M. Tsantaki ${ }^{1}$, B. Rojas-Ayala ${ }^{1}$, and V. Neves ${ }^{3}$ \\ 1 Instituto de Astrofísica e Ciências do Espaço, Universidade do Porto, CAUP, Rua das Estrelas, 4150-762 Porto, Portugal \\ e-mail: daniel.andreasen@astro.up.pt \\ 2 Departamento de Física e Astronomia, Faculdade de Ciências, Universidade do Porto, Rua Campo Alegre, 4169-007 Porto, Portugal \\ 3 Departamento de Física, Universidade Federal do Rio Grande do Norte, 59072-970 Natal, RN, Brazil
}

Received 4 September 2015 / Accepted 28 October 2015

\section{ABSTRACT}

\begin{abstract}
Context. Effective temperature, surface gravity, and metallicity are basic spectroscopic stellar parameters necessary to characterize a star or a planetary system. Reliable atmospheric parameters for FGK stars have been obtained mostly from methods that rely on high resolution and high signal-to-noise optical spectroscopy. The advent of a new generation of high resolution near-infrared (NIR) spectrographs opens the possibility of using classic spectroscopic methods with high resolution and high signal-to-noise in the NIR spectral window.

Aims. We compile a new iron line list in the NIR from a solar spectrum to derive precise stellar atmospheric parameters, comparable to the ones already obtained from high resolution optical spectra. The spectral range covers $10000 \AA$ to $25000 \AA$, which is equivalent to the $Y, J, H$, and $K$-bands.

Methods. Our spectroscopic analysis is based on the iron excitation and ionization balance done in local thermodynamic equilibrium. We use a high resolution and high signal-to-noise ratio spectrum of the Sun from the Kitt Peak telescope as a starting point to compile the iron line list. The oscillator strengths $(\log g f)$ of the iron lines were calibrated for the Sun. The abundance analysis was done using the MOOG code after measuring equivalent widths of 357 solar iron lines.

Results. We successfully derived stellar atmospheric parameters for the Sun. Furthermore, we analysed HD 20010, a F8IV star, from which we derived stellar atmospheric parameters using the same line list as for the Sun. The spectrum was obtained from the CRIRESPOP database. The results are compatible with the values found in the literature, confirming the reliability of our line list. However, we obtain large errors due to the quality of the data.
\end{abstract}

Key words. Sun: atmosphere - stars: solar-type - techniques: spectroscopic - stars: fundamental parameters stars: individual: HD 20010

\section{Introduction}

Effective temperature $\left(T_{\text {eff }}\right)$, surface gravity $(\log g)$, and metallicity $([\mathrm{M} / \mathrm{H}]$, where iron is normally used as a proxy) are fundamental atmospheric parameters necessary to characterise a single star, and to determine other indirect fundamental parameters such as mass, radius, and age from stellar evolutionary models (see e.g. Girardi et al. 2000; Dotter et al. 2008; Baraffe et al. 2015). Precise and accurate stellar parameters are also essential in exoplanet searches. Planetary radius and mass are mainly found from lightcurve analysis and radial velocity analysis, respectively. The determination of the mass of the planet implies a knowledge of the stellar mass, while the measurement of the radius of the planet is dependent on our capability to derive the radius of the star (see e.g. Torres et al. 2008; Ammler-von Eiff et al. 2009; Torres et al. 2012).

The derivation of precise stellar atmospheric parameters is not a simple task. Different approaches often lead to discrepant results (see e.g. Santos et al. 2013). Interferometry is usually considered an accurate method for deriving stellar radii (e.g. Boyajian et al. 2012); however, it is only applicable for bright nearby stars. Asteroseismology, on the other hand, reveals the

* Full Table 1 is only available at the CDS via anonymous ftp to cdsarc.u-strasbg.fr (130.79.128.5) or via

http://cdsarc.u-strasbg.fr/viz-bin/qcat?J/A+A/585/A143 inner stellar structure by observing the stellar pulsations at the surface. From asteroseismology it is possible to measure the surface gravity and mean density, and therefore to calculate the mass and radius (e.g. Kjeldsen \& Bedding 1995).

A crucial parameter for the indirect determination of stellar bulk properties is the effective temperature. In that respect, the infrared flux method (IRFM) has proven to be reliable for FGK dwarf and subgiant stars. However, the IRFM needs a priori knowledge of the bolometric flux, reddening, surface gravity, and stellar metallicity (Blackwell \& Shallis 1977; Ramírez \& Meléndez 2005; Casagrande et al. 2010).

Finally, the use of high resolution spectroscopy along with stellar atmospheric models is an extensively tested method that allows the derivation of the fundamental parameters of a star (see e.g. Santos et al. 2013; Valenti \& Fischer 2005). The procedure depends on the quality of the spectra, their resolution, and wavelength region. For low resolution spectra $(\lambda / \Delta \lambda<20000)$ the preferred method is to fit the overall observed spectrum with a synthetic one (see e.g. Recio-Blanco et al. 2006). Higher resolution spectra of slowly rotating stars (below 10 to $15 \mathrm{~km} \mathrm{~s}^{-1}$ ) are in the regime where the equivalent width (EW) method can be used (for details see Sect. 2).

The derivation of stellar atmospheric parameters from high resolution spectra in the optical is now based on a standard procedure (see e.g. Valenti \& Fischer 2005; Sousa et al. 2008). With the advancement of high resolution near-infrared 
(NIR) instruments, we will now be able to use a similar technique to that used in the optical part of the spectrum (see e.g. Meléndez \& Barbuy 1999; Sousa et al. 2008; Tsantaki et al. 2013; Mucciarelli et al. 2013; Bensby et al. 2014). At the moment, the GIANO spectrograph installed at Telescopio Nazionale Galileo (TNG) is already available (Origlia et al. 2014), as is the infrered doppler instrument (IRD) installed at the Subaru telescope (Kotani et al. 2014). Three new spectrographs are planned for the near future: 1) Calar Alto high-Resolution search for M dwarfs with Exoearths with Near-infrared and optical Échelle Spectrographs (CARMENES) for the $3.5 \mathrm{~m}$ telescope at Calar Alto Observatory (Quirrenbach et al. 2014) had first light in December 2014; 2) the CRyogenic InfraRed Echelle Spectrograph Upgrade Project (CRIRES+) at the Very Large Telescope (VLT; Follert et al. 2014) with expected first light in 2017; and 3) un SpectroPolarimètre Infra-Rouge (SPIRou, A Near-InfraRed Spectropolarimeter) at the Canada-FranceHawaii Telescope (CFHT; Delfosse et al. 2013; Artigau et al. 2014) with expected first light in 2017 as well. The spectral resolutions for these spectrographs range between 50000 and 100000 .

Even though reliable line lists for the derivation of stellar parameters using optical spectra exist, the situation is different in the NIR regime. There are a few for the synthesis method (e.g. Önehag et al. 2012; Origlia et al. 2013; Rhodin 2015), and there is the large general compilation by Meléndez \& Barbuy (1999). Moreover, there are line lists compiled in the NIR for the Apache Point Observatory Galactic Evolution Experiment (APOGEE) survey (see e.g. Smith et al. 2013; Shetrone et al. 2015). Thus, in this paper we want to explore the possibility of creating a line list of iron lines in the NIR which can be applied for FGKM stars optimized for the EW method in a consistent way, as is currently done for these stars in the optical (FGK only). The paper is organized as follows. In Sect. 2 we present how to compile a line list and the method for deriving parameters with the equivalent width method for an iron line list. In Sect. 3 we present the results for the derived parameters for the Sun and HD 20010. In Sect. 4 we discuss our results.

\section{Method}

The two most widely used methods for deriving stellar atmosphere parameters from a spectrum are spectral synthesis and the equivalent width (EW) method. The spectral synthesis method compares synthetic spectra to an observed spectrum and finds the best model by a minimization procedure (see e.g. Valenti \& Fischer 2005; Önehag et al. 2012; Blanco-Cuaresma et al. 2014). When the minimization procedure reaches a minimum, the final atmospheric parameters are found.

The EW method (see e.g. Sousa et al. 2008; Mucciarelli et al. 2013; Bensby et al. 2014), which we use in this work, is based on the measurements of EWs from a list of lines combined with the matching atomic data. The EW for a single line is given as

$E W=\int_{0}^{\infty}\left(1-\frac{F_{\lambda}}{F_{0}}\right) \mathrm{d} \lambda$,

where $F_{0}$ is the continuum level and $F_{\lambda}$ is the flux as a function of wavelength.

Using this method, we obtain the abundance of individual lines by the radiative transfer code MOOG (Sneden 1973, version 2013) under the assumption of local thermodynamic equilibrium (LTE). To obtain metallicity, we expect every spectral line of the same element to produce the same abundance. In our analysis, we use neutral iron (Fe I) and single ionized iron (Fe II) as a proxy for the metallicity. The effective temperature and surface gravity are derived from the principles of ionization and excitation equilibrium (see Gray 2005).

A disadvantage of the EW method may be a miscalculation of the EW. For example, the placement of the continuum level may be incorrect, which leads to an over- or underestimation of the EW for the given line. Another source of error is contamination with either telluric lines or other neighbouring lines. The relative error is typically larger for the weak lines. In this work we will focus on the spectral region covered by the $Y, J, H$, and $K$-bands, which covers more than $15000 \AA$.

\subsection{Compiling the line list}

To compile the line list we used the Vienna Atomic Line Database (VALD3; Piskunov et al. 1995; Kupka et al. 2000). First, we downloaded a list of all iron lines present in the near infrared region covering $10000 \AA$ to $25000 \AA$. In total, 78537 iron lines were found in this spectral region (50198 Fe I lines and 28339 Fe II lines). Many of these lines are too faint to be detected in a spectrum of a solar-type star. A spectrum of the Sun was downloaded from the BASS2000 web page ${ }^{1}$ to select the best lines for this analysis. The NIR part of the spectrum was obtained from the Kitt Peak telescope (Hinkle et al. 1995) at a resolution of $0.004 \AA$ at $10000 \AA$ to $0.1 \AA$ at $50000 \AA$. The spectrum was downloaded in the highest possible resolution at a given wavelength. The signal-to-noise ratio of the spectrum varies from 3000 at $12000 \AA$ down to 1400 at $21400 \AA$.

We use the Automatic Routine for line Equivalent widths in stellar Spectra (ARES) software ${ }^{2}$ (Sousa et al. 2007, 2015) to automatically measure the EWs of all the lines. Since the first version of ARES expects a 1D spectrum with equidistant wavelength spacing, the solar spectrum was interpolated to a regular grid with constant wavelength step of $0.01 \AA$. This did not change the appearance of the spectrum, or therefore the EW. The EWs are measured by fitting Gaussian profiles to spectral lines. For a given line, ARES outputs the central wavelength of the line, the number of lines fitted for the final result, the depth of the line, the FWHM of the line, the EW of the line, and the Gaussian coefficients for the line.

Once this step was done we then selected a subset of lines using the following criteria:

- If the number of fitted lines by ARES for a given line is higher than 10, this line is rejected because it is believed to be severely blended.

- If the EW is lower than $5 \mathrm{~m} \AA$ for an absorption line, the strength is too low and it may be difficult to see the line in spectra with low signal-to-noise ratio or a spectrum with many spectral features.

- If the EW is higher than $200 \mathrm{~m} \AA$ for a given line, the strength is too high and we can no longer fit the line with a Gaussian profile since the absorption line no longer has a pure Gaussian profile.

- If the fitted central wavelength is more than $0.05 \AA$ from the wavelength provided by VALD3, the line will also be rejected to avoid false identification.

\footnotetext{
1 The web page can be found here: bass2000.obspm.fr/solar spect.php

2 The ARES software can be found here: http://www.astro.up. $\mathrm{pt} / \sim$ sousasag/ares/. The following settings were used: lambdai = 7500 , lambdaf $=54000$, smoothder $=4$, space $=2.0$, rejt $=0.995$, lineresol $=0.07$, and miniline $=2$.
} 
After the automatic removal of lines following the above criteria we reduced the number of lines to 6060 and 2735 for Fe I and Fe II, respectively.

\subsection{Visual removal of lines}

A visual inspection of the lines is necessary at this point in order to allow us to select only the best lines. We consider the best lines to be the ones that are not blended, and therefore reliable EW measurements can be made.

In this step we analyzed in detail small spectral windows ( $3 \AA$ in width) around each line. For each spectral window, the corresponding absorption lines for all elements were downloaded from the VALD3 database. The location of these lines were plotted on top of the solar spectrum, and any iron line was excluded if a line of another element was present at the same wavelength. Iron lines were also excluded when the absorption line was severely blended by other spectral lines. Many of the removed iron lines at this step have higher excitation potential than the final line list since these lines are generally weaker than those with lower excitation potential. After this step 593 Fe I lines and $22 \mathrm{Fe}$ II lines remained in the sample.

For some spectral regions it was not clear which element or elements caused an absorption line. In these cases the iron lines were marked for further investigation with the synthesis explained below.

\subsection{Synthesis of selected lines}

Lines from all elements in a $6 \AA$ window around an iron line marked for further investigation were used to make a synthetic spectrum. The synthetic spectra were made with MOOG with the synth driver. We use an ATLAS9 atmosphere model (Kurucz 1993) with the nominal solar atmospheric parameters $T_{\text {eff }}=$ $5777 \mathrm{~K}, \log g=4.438$, and $\xi_{\text {micro }}=1.0 \mathrm{~km} \mathrm{~s}^{-1}$ to resemble the Sun. We used three different iron abundances for the synthesis. The first with solar iron abundance, the second with 0.2 dex above solar, and the third with 0.2 dex below solar. We consider a solar iron abundance of 7.47 as presented in Gonzalez \& Laws (2000). This choice of solar parameters and iron abundances was done to match the values used by our team in previous papers (see e.g. Santos et al. 2013, and references therein) and thereby to provide consistency within our group. If the synthetic spectra shows variation at the absorption line of interest with respect to the different iron abundances, then it is likely to be an iron line. We also changed abundances of other elements in the proximity to see if our line is blended with other elements. An example of these plots can be seen in Fig. 1.

Sometimes more than one iron line might be present with very similar wavelengths so they can no longer be resolved. In order to find the iron line that is creating the observed absorption line, one of the two were excluded from the line list for the synthetic spectra. If this removed (either fully or partially) the absorption line in the synthetic spectra, then it was considered the cause for the observed absorption line, otherwise we excluded the line from the line list presented in this work.

A few times two iron lines had identical wavelengths and excitation potential. In those cases the $\log g f$ were combined (sum of the $g f$-value) to create a single line that can be analyzed with our method. We ended up with 414 and 12 lines of Fe I and Fe II, respectively.
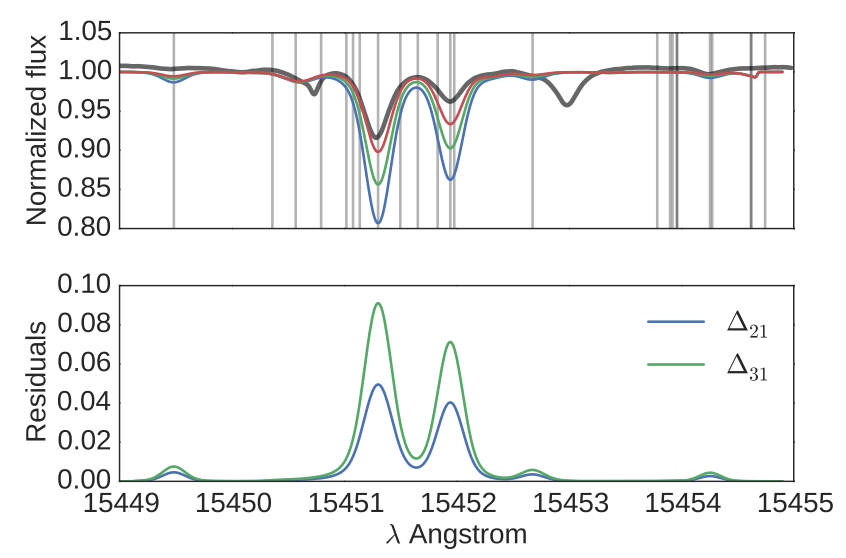

Fig. 1. Top panel: observed spectra in grey, while the coloured curves are synthetic spectra with increasing iron abundance as the two central lines get deeper. The iron abundance varies by a total of 0.4 dex. The vertical lines show all the places where there are iron lines in the line list. Bottom panel: the two curves show the difference between the first synthetic spectrum and the second $\left(\Delta_{21}\right)$ and between the first synthetic spectrum and the third $\left(\Delta_{31}\right)$; this highlights where the change in iron abundance has an impact.

\subsection{Calibrating the line list: astrophysical log gf values}

The iron abundances for each line were calculated using the same solar atmosphere model as described above for synthesis. This step allowed us to remove possible outliers based on the assumption that errors in the $\log g f$ values from the VALD3 database would never lead to variations of the derived iron abundance of more than 1 dex. All the Fe I lines before recalibration of the oscillator strength and removal of lines which deviates more than 1 dex are presented in Fig. A.1. We note that we only removed Fe I lines here because the Fe II lines were sparse and essential to determining the surface gravity when we reach ionization balance, as explained in Sect. 2.6. After removal of $1 \mathrm{dex}$ outliers we were down to 319 and 12 lines, for Fe I and Fe II respectively.

After the removal of lines from the complete VALD3 line list we recalibrated the oscillator strength of the lines $(\log g f)$ in order to match the adopted solar abundance, an inverse solar analysis. This allowed us to perform a differential analysis other stars. Similar approaches have been done by Sousa et al. (2008), Önehag et al. (2012), Rhodin (2015). In Fig. 2 the EWs of the iron lines present in the Sun are plotted as a function of the excitation potential. This plot shows the distribution after recalibration of $\log g f$ after the cut for lines with abundances deviating more than $1 \mathrm{dex}$ from the solar value. The majority of the iron lines are found in $\mathrm{H}$-band as shown in Fig 3.

\subsection{Removal of high dispersion lines}

To chose which line-derived abundances are less prone to errors caused by the uncertainties in the EW measured, we decided to do the following test. A Gaussian distribution was made for the EW of each line. We used the width for the Gaussian distribution following the formula presented in Cayrel (1988),

$\sigma \simeq 1.6 \frac{\sqrt{\Delta \lambda E W}}{S / N}$ 


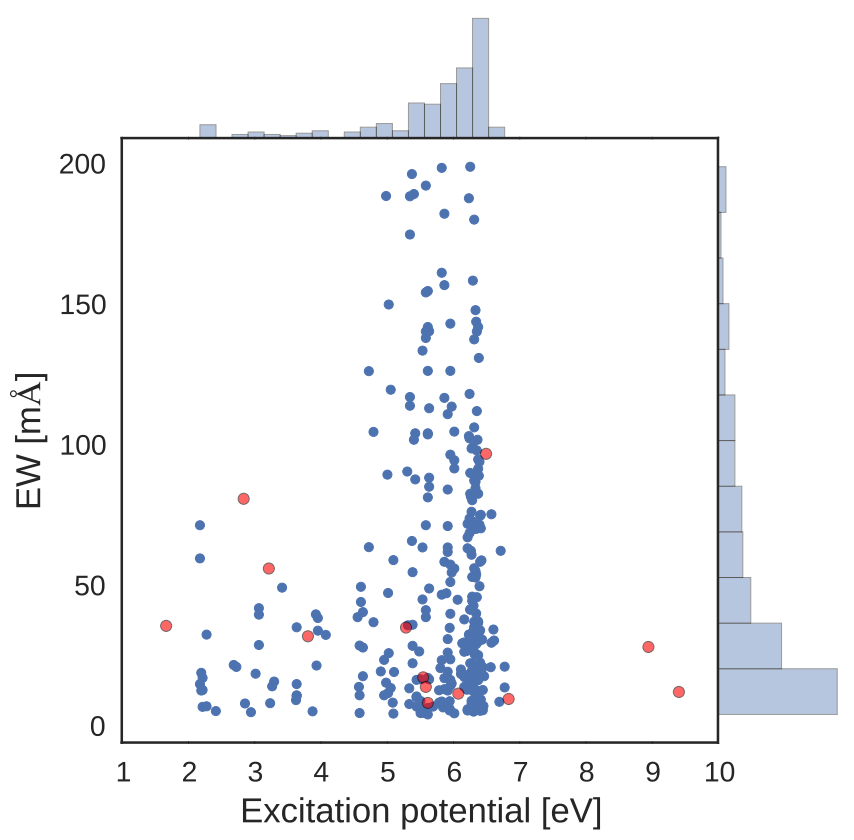

Fig. 2. Distribution of Fe I and Fe II lines in blue and red, respectively. The distribution shows the measured EWs for the Sun as a function of the excitation potential.

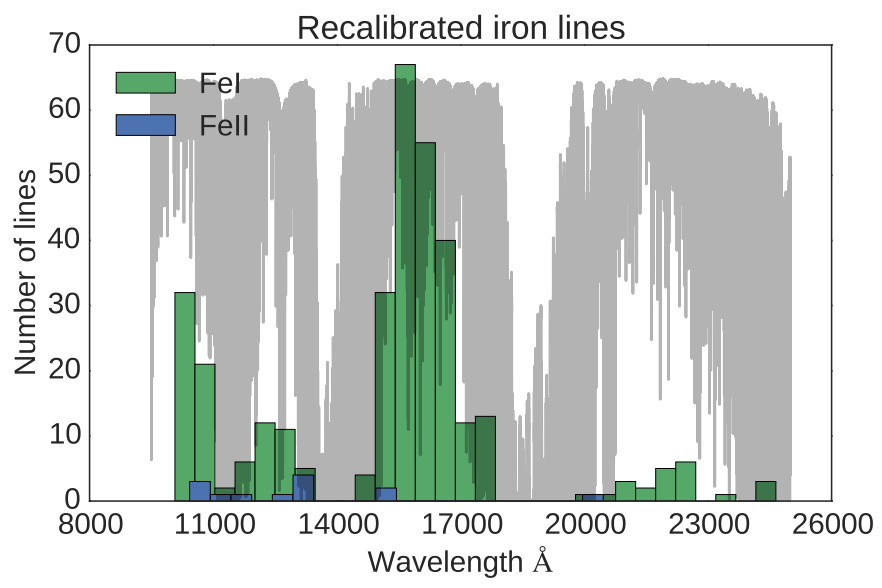

Fig. 3. Distribution of both Fe I and Fe II lines plotted on top of the solar spectrum. The distributions are for the final line list. There are two areas in the spectrum with high telluric contamination, which also mark the border between the filters we use from $J$ to $H$ around $14000 \AA$ and from $H$ to $K$ around $19000 \AA$. Most of the lines are located in the $H$-band.

where $\Delta \lambda=0.1 \AA$ and we considered a signal-to-noise ratio of 50, much lower than the signal-to-noise ratio of the spectrum. This width was used to create a Gaussian distribution with a mean around the original EW:

$f(x, E W, \sigma)=\frac{1}{\sqrt{2 \pi \sigma^{2}}} \mathrm{e}^{-\frac{(x-E W)^{2}}{2 \sigma^{2}}}$.

We made 100 draws for each line and derived the abundance with solar parameters, using the same atmospheric model as described above. For each line we calculated the mean absolute deviation (MAD). The MAD values are plotted against the original EWs in the upper part of Fig. 4. We see a clear trend towards weaker lines, which is expected since a small absolute change in the EW results in a large relative change in abundance, hence a high MAD value. However, this does not mean that the abundances of these lines have a high dispersion. Therefore, we

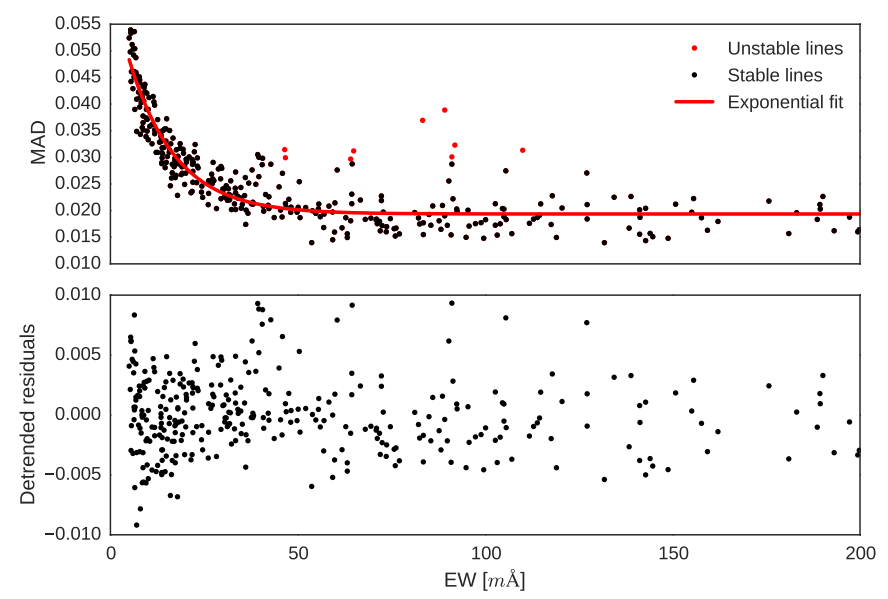

Fig. 4. Upper plot: MAD values of 100 noisy lines with a simulated signal-to-noise ratio of 50 . The red points are the 23 detected unstable lines, and the red curve is the last fit in the iterative removal of unstable lines. Lower plot: detrended points from the upper plot, used for the $3 \sigma$ calculation for each iteration

detrended the data with a fitted exponential. The residuals are shown in the lower part of Fig. 4. We use the detrended data as a measurement for the dispersion of a given line. A single point above $3 \sigma$ is then removed iteratively until there are no longer any points above this threshold. In this process we remove 33 lines. The final line list is presented in Table 1.

\subsection{Deriving parameters with the EW method}

Once the EWs have been measured for all iron lines in the line list (or for as many as possible), the next step is to derive the atmospheric parameters. Atmosphere models are necessary for computing abundances of the lines. The literature offers the possibility to choose from a wide variety of model atmospheres. Models like ATLAS9 (Kurucz 1993) and MARCS (Gustafsson et al. 2008) have been the preferred atmosphere models for the derivation of spectroscopic parameters for FGK stars.

We use the ATLAS9 models which, for efficiency, are created in a grid according to effective temperature, surface gravity, and metallicity. In order to search for final parameters it is necessary to interpolate models from the grid, thus allowing a finer grid space to be examined (see e.g. Sousa 2014). This grid of atmosphere models has been used extensively by our group; it allows us to work consistently over multiple wavelength regions (optical and NIR).

For a given atmosphere model, abundances of all the lines in the line list are calculated. By removing any correlation between the excitation potential and abundance of all lines (from the same element) the effective temperature is constrained. In a similar way, the microturbulence can be constrained by removing any correlation between the reduced EW $(\log E W / \lambda)$ and iron abundances, and the surface gravity is found when there is ionization balance, i.e. the mean abundance of Fe I and Fe II are equal. Lastly, the iron abundance comes from calculating the mean of all the iron abundances. When there is no longer any correlation, the final atmospheric parameters are obtained from the last atmosphere model.

In order to find the best atmosphere model, a minimization algorithm is used based on the downhill simplex method (Press et al. 1992), which searches the parameter space for the best fitting atmospheric model, i.e. the best parameters. The 
Table 1. Final line list as found for the Sun with astrophysical $\log g f$ values.

\begin{tabular}{|c|c|c|c|c|}
\hline Wavelength [Å] & Element & $E P[\mathrm{eV}]$ & $\log g f$ & $E W[\mathrm{~m} \AA]$ \\
\hline 10070.521 & Fe I & 5.51 & -1.527 & 6.6 \\
\hline 10080.415 & $\mathrm{Fe} I$ & 5.10 & -2.008 & 5.3 \\
\hline 10081.394 & $\mathrm{Fe} I$ & 2.42 & -4.551 & 6.2 \\
\hline 10137.100 & $\mathrm{Fe} I$ & 5.09 & -1.768 & 9.2 \\
\hline 10142.844 & $\mathrm{Fe} I$ & 5.06 & -1.574 & 14.4 \\
\hline 10155.163 & $\mathrm{Fe} I$ & 2.18 & -4.340 & 15.8 \\
\hline 10156.507 & $\mathrm{Fe} I$ & 4.59 & -2.125 & 11.8 \\
\hline 10167.469 & $\mathrm{Fe} I$ & 2.20 & -4.199 & 19.8 \\
\hline 10195.106 & $\mathrm{Fe} I$ & 2.73 & -3.625 & 21.9 \\
\hline 10227.991 & $\mathrm{Fe} I$ & 6.12 & -0.449 & 19.4 \\
\hline 10230.796 & $\mathrm{Fe} I$ & 6.12 & -0.414 & 21.0 \\
\hline 10265.218 & $\mathrm{Fe} I$ & 2.22 & -4.668 & 7.7 \\
\hline 10327.340 & $\mathrm{Fe} I$ & 5.54 & 0.504 & 134.4 \\
\hline 10332.328 & $\mathrm{Fe} I$ & 3.63 & -3.145 & 10.1 \\
\hline 10340.886 & $\mathrm{Fe}_{\mathrm{I}}$ & 2.20 & -3.672 & 46.7 \\
\hline 10347.966 & $\mathrm{Fe}_{\mathrm{I}}$ & 5.39 & -0.754 & 36.9 \\
\hline 10353.805 & $\mathrm{Fe} I$ & 5.39 & -1.035 & 23.2 \\
\hline 10364.063 & $\mathrm{Fe} I$ & 5.45 & -1.129 & 17.3 \\
\hline 10379.000 & $\mathrm{Fe}_{\mathrm{I}}$ & 2.22 & -4.246 & 18.0 \\
\hline 10388.746 & $\mathrm{Fe} I$ & 5.45 & -1.527 & 7.8 \\
\hline$\ldots$ & $\ldots$ & & & $\ldots$ \\
\hline 10427.305 & $\mathrm{Fe}$ II & 6.08 & -1.662 & 12.4 \\
\hline 10501.498 & $\mathrm{Fe}$ II & 5.55 & -1.926 & 18.2 \\
\hline 10862.644 & $\mathrm{Fe}$ II & 5.59 & -2.043 & 14.8 \\
\hline 11125.580 & Fe II & 5.62 & -2.301 & 9.1 \\
\hline 11833.056 & $\mathrm{Fe}$ II & 2.84 & -3.379 & 81.7 \\
\hline 12913.876 & Fe II & 6.50 & 0.045 & 97.7 \\
\hline 13251.144 & $\mathrm{Fe}$ II & 9.41 & 0.860 & 13.0 \\
\hline 13277.306 & Fe II & 5.29 & -2.043 & 35.9 \\
\hline 13294.853 & $\mathrm{Fe}$ II & 3.22 & -3.613 & 56.9 \\
\hline 13419.109 & Fe II & 3.81 & -3.484 & 32.8 \\
\hline 15247.133 & $\mathrm{Fe}$ II & 6.84 & -1.691 & 10.5 \\
\hline 15350.156 & $\mathrm{Fe}$ II & 8.95 & 0.602 & 29.0 \\
\hline 20460.070 & $\mathrm{Fe}$ II & 1.67 & -5.758 & 36.5 \\
\hline
\end{tabular}

Notes. A complete version of this table is available at the CDS.

convergence criteria for the correlation between excitation potential and abundances is a slope lower than 0.001. A slope lower than 0.002 for the correlation between the reduced EW and the abundances, and a difference of less than 0.005 between the mean abundances for Fe I and Fe II is used in e.g. Sousa et al. (2008) and Tsantaki et al. (2013).

The error estimate is based on the same method presented in Gonzalez \& Vanture (1998). The uncertainty on $\xi_{\text {micro }}$ is determined from the standard deviation in the slope of abundance versus reduced equivalent width; the uncertainty on $T_{\text {eff }}$ is determined from the uncertainty on the slope of abundance versus excitation potential in addition to the uncertainty on $\xi_{\text {micro }}$; the uncertainty on the iron abundance is a combination of the uncertainties on $T_{\text {eff }}, \xi_{\text {micro }}$, and the scatter of the individual Fe I abundances. The uncertainty on the surface gravity is based on the uncertainty on $T_{\text {eff }}$ and the scatter in Fe II abundances.

\section{Results}

\subsection{Derived parameters for the Sun}

We derived the stellar atmospheric parameters for the Sun using the resulting line list (including the solar calibrated astrophysical
Table 2. The derived parameters for the Sun at different signal-to-noise ratios.

\begin{tabular}{lllll}
\hline \hline$S / N$ & $T_{\text {eff }}(\mathrm{K})$ & $\log g(\mathrm{dex})$ & {$[\mathrm{Fe} / \mathrm{H}](\mathrm{dex})$} & $\xi_{\text {micro }}\left(\mathrm{km} \mathrm{s}^{-1}\right)$ \\
\hline Original & $5776 \pm 0$ & $4.43 \pm 0.00$ & $0.00 \pm 0.00$ & $0.99 \pm 0.00$ \\
\hline 25 & $5808 \pm 119$ & $4.50 \pm 0.20$ & $0.00 \pm 0.06$ & $1.28 \pm 0.51$ \\
50 & $5780 \pm 41$ & $4.45 \pm 0.09$ & $0.00 \pm 0.02$ & $1.06 \pm 0.26$ \\
100 & $5776 \pm 22$ & $4.44 \pm 0.03$ & $0.00 \pm 0.01$ & $1.02 \pm 0.10$ \\
150 & $5776 \pm 12$ & $4.44 \pm 0.02$ & $0.00 \pm 0.00$ & $1.01 \pm 0.09$ \\
225 & $5779 \pm 10$ & $4.44 \pm 0.01$ & $0.00 \pm 0.00$ & $1.01 \pm 0.07$ \\
300 & $5777 \pm 10$ & $4.43 \pm 0.02$ & $0.00 \pm 0.00$ & $1.01 \pm 0.06$ \\
\hline
\end{tabular}

Notes. The error is the $3 \sigma$ standard deviation calculated from the ten runs at each signal-to-noise ratio.

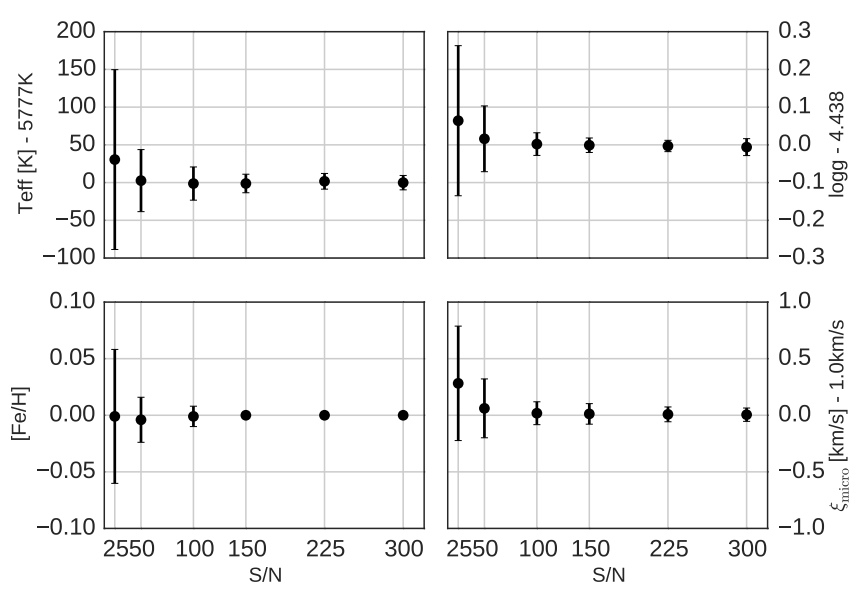

Fig. 5. Derived parameters as a function of the signal-to-noise. The error is the $3 \sigma$ standard deviation from the ten different runs for each signalto-noise. The upper left plot shows the effective temperature. The upper right plot show the surface gravity $(\log g)$. The lower left shows the iron abundance, used as a proxy for the metallicity. Finally, in the lower right plot the microturbulence is shown.

$\log g f$ values). We used the minimization procedure described in Sect. 2.6. Since the line list and $\log g f$ values were selected using the solar spectrum, it is with no surprise that the derived parameters for the Sun perfectly match the adopted solar values within the error bars as seen in Table 2 .

Moreover, we derived parameters for different signal-tonoise ratios, namely $25,50,100,150,225$, and 300 . The signalto-noise ratios were obtained by drawing EW from a Gaussian distribution with widths dependent on the EW itself and the signal-to-noise as described above in Sect. 2.5. For each considered signal-to-noise, we made ten random line lists, giving us a total of 60 line lists. This exercise shows the expected precision for different signal-to-noise ratios with the proposed line list. The final results are presented in Fig. 5. The error bars represent the $3 \sigma$ standard deviation from the ten different runs. As seen from the figure, we expect to derive precise parameters (effective temperature more precise than $50 \mathrm{~K}$, surface gravity with a precision of $0.1 \mathrm{dex}$, iron abundance with a precision of 0.05 , and microturbulence with a precision of 0.3 ) down to a signal-tonoise ratio of 50. At higher signal-to-noise ratios the precision increases. For example at a signal-to-noise ratio of 100 the errors are reduced by a factor of 2 . The results can also be seen in Table 2. This shows that the line list is fully reliable for the whole 
Table 3. Selection of literature values for the atmospheric parameters for HD 20010.

\begin{tabular}{l|llll}
\hline \hline Author & $T_{\text {eff }}(\mathrm{K})$ & $\log g(\mathrm{dex})$ & {$[\mathrm{Fe} / \mathrm{H}](\mathrm{dex})$} & $\xi_{\text {micro }}\left(\mathrm{km} \mathrm{s}^{-1}\right)$ \\
\hline Balachandran (1990) & 6152 & 4.15 & $-0.27 \pm 0.08$ & 1.6 \\
Favata et al. (1997) & 6000 & $\ldots$ & $-0.35 \pm 0.07$ & $\ldots$ \\
Santos et al. (2004) & $6275 \pm 57$ & $4.40 \pm 0.37$ & $-0.19 \pm 0.06$ & $2.41 \pm 0.41$ \\
Gonzalez et al. (2010) & $6170 \pm 35$ & $3.93 \pm 0.02$ & $-0.206 \pm 0.025$ & $1.70 \pm 0.09$ \\
Ramírez et al. (2012) & $6073 \pm 78$ & $3.91 \pm 0.03$ & $-0.30 \pm 0.05$ & $\ldots$ \\
Mortier et al. (2013) & 6114 & $\ldots$ & -0.19 & $\ldots$ \\
\hline Mean & $6131 \pm 255$ & $4.01 \pm 0.60$ & $-0.23 \pm 0.14$ & $1.90 \pm 1.08$ \\
\hline
\end{tabular}

Notes. The mean and a $3 \sigma$ standard deviation is presented at the end of the table from the literature values included, which we use as a reference for our derived parameters.

range of signal-to-noise ratios considered, even if the precision decreases at lower signal-to-noise ratios as expected.

\subsection{Derived parameters for HD 20010}

To test our new line list we search for a well-studied solartype star. The spectrum for such a target needs to be available in the NIR at both high resolution and high signal-to-noise. An ideal place to look for such a star is the CRIRES-POP database (Lebzelter et al. 2012). Here, the best target for testing is HD 20010, an F8 subgiant star. This star has been part of many surveys and is therefore well studied. Different parameters from the literature are listed in Table 3.

The data available at CRIRES-POP are in the raw format and pipeline reduced, while three small pieces of the spectra are fully reduced on the web page ${ }^{3}$. The data is in the standard CRIRES format with each fits file including four binary tables with the data from the four detectors. In the future, the final reduced data will be presented by the CRIRES-POP team. In contrast to the pipeline reduced data, this will be of higher quality, a better wavelength calibration, and telluric correction. We measured the EWs of the pipeline reduced spectra, and where there was an overlap with the fully reduced spectrum, we measured both as a consistency check. The measured EWs from the fully reduced spectra were consistent with the measured EWs from the pipeline reduced spectra. As mentioned above, we use the $Y, J, H$, and $K$-bands which are all available for this star. The spectra come in pieces of $50 \AA$ to $120 \AA$. These pieces overlap each other, and we were able to measure the EW for a single line up to five times. Unfortunately, wavelength calibration is a difficult task for CRIRES owing to the rather small spectral regions measured on each detector. Each calibration was performed separately for each detector and required the availability of a sufficient number of calibration lines in the respective spectral region. This was not always the case and a default linear solution was applied. A pipeline reduced spectrum shows up as a stretched spectrum if the wavelength calibration is poor compared to a model spectrum or a solar spectrum, for example. The wavelength calibration does not have any effect on the signal-to-noise ratio, which is generally high for the spectrum of HD 20010. The signal-to-noise varies between 200 and 400 for different chunks. The pipeline reduced spectra for HD 20010 contains tellurics and the wavelength is shifted in radial velocity. All of these factors make the line identification very difficult,

\footnotetext{
3 http://www.univie.ac.at/crirespop/data.htm
}

and so we developed a program ${ }^{4}$ to properly identify the lines, which does the following:

1. Plotting the observed spectrum;

2. Overplotting a model spectrum. In this particular case the solar spectrum was used since the atmospheric parameters are close enough, so the sun was able to serve as a model;

3. Overplotting a telluric spectrum from the TAPAS web page ${ }^{5}$ (Bertaux et al. 2014);

4. Overplotting vertical lines at the location of lines in the list;

5. Calculating the cross-correlation function (CCF) for the telluric spectrum with respect to the observed spectrum, locating the maximum value by a Gaussian fit, and using this to shift the telluric spectrum with the found RV;

6. Performing the same as step 5, but for the model;

7. Shifting the lines with the same RV as found for the model/solar spectrum.

The final plot shows the shifted spectra, and the CCFs at the sides. An example of the software in use is shown in Fig. 6. The two RVs are part of the title of the plot.

Once the lines were identified, the EWs were measured with the splot routine in Image Reduction and Analysis Facility (IRAF). The reason not to choose ARES for this task was to visually confirm the identification of the line given the relative poor wavelength calibration. We were able to measure $249 \mathrm{Fe}$ I lines and $5 \mathrm{Fe}$ II lines compared to $344 \mathrm{Fe}$ I lines and 13 Fe II lines for the Sun over the whole NIR spectral region. Whenever we had more than one measurement of a line, the average was used for the final EW.

We derived the stellar parameters using the standard procedure (see Sect. 2.6) as done for the Sun. Given the relatively low quality of the spectrum of HD 20010 (see below) and because it is not corrected for telluric contamination, we made a cut in EW at $5 \mathrm{~m} \AA$ in order to remove the lines which are most affected by contamination from either telluric or other line blends. Additionally, we made a cut in EP at $5.5 \mathrm{eV}^{6}$ because the $\mathrm{Fe}$ I and Fe II lines usually used for stellar parameter determination in the optical regime are also limited to similar values (see e.g. Sousa et al. 2008). Higher excitation potential lines are also more likely to be affected by non-LTE effects. When deriving the

4 The program (plot_fits) is open source and can be found here: https://github.com/DanielAndreasen/astro_scripts

5 http://ether.ipsl.jussieu.fr/tapas/

6 We also derived the stellar parameters without any cut in the EP, but the resulting values were always overestimated (e.g. fixing $\log g$ to 4.01 we obtained a temperature of $6660 \mathrm{~K}$ and metallicity of $+0.19 \mathrm{dex}$ ). 

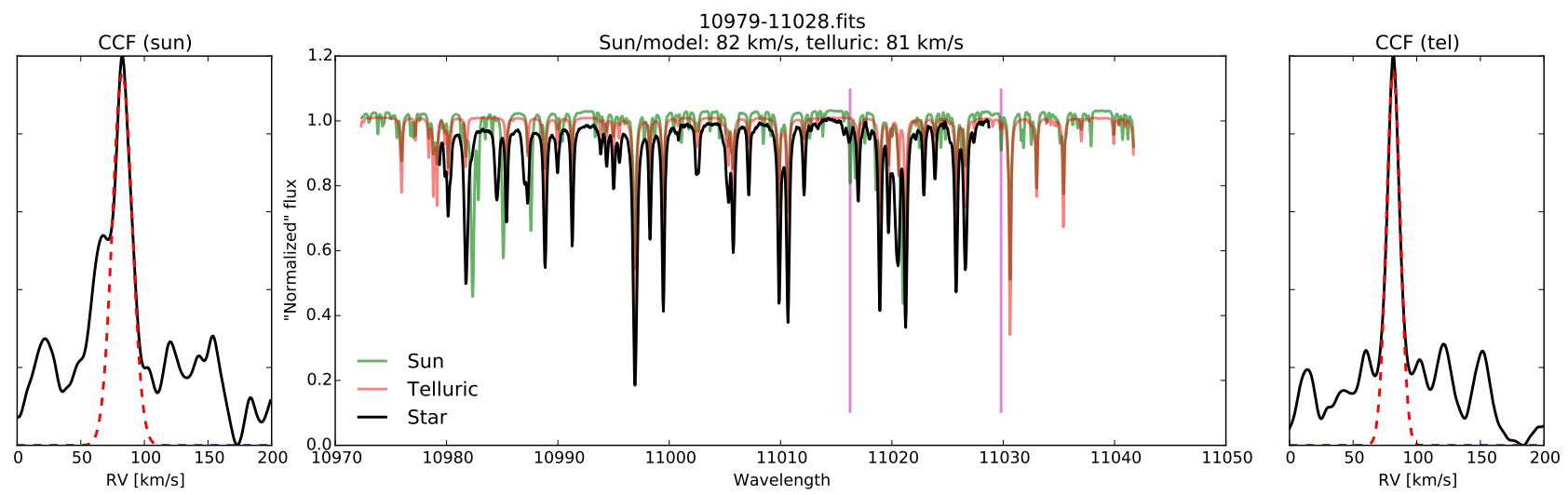

Fig. 6. Middle plot: piece of HD 20010 (black), the model spectrum, in this case the Sun (green), a telluric spectrum (red), and two lines from our line list (magenta vertical lines). Left plot: CCF of the Sun with a fitted Gaussian. Right plot: same as the one to the left, but for the telluric spectrum.

Table 4. Derived parameters for HD 20010 with and without fixed surface gravity cut after $3 \sigma$ outlier removal.

\begin{tabular}{lllll}
\hline \hline & $T_{\text {eff }}(\mathrm{K})$ & $\log g(\mathrm{dex})$ & $\xi_{\text {micro }}\left(\mathrm{km} \mathrm{s}^{-1}\right)$ & {$[\mathrm{Fe} / \mathrm{H}](\mathrm{dex})$} \\
\hline Literature & $6131 \pm 255$ & $4.01 \pm 0.60$ & $1.90 \pm 1.08$ & $-0.23 \pm 0.14$ \\
\hline $6116 \pm 224$ & $4.21 \pm 0.58$ & $2.45 \pm 0.45$ & $-0.14 \pm 0.14$ \\
$6144 \pm 212$ & 4.01 (fixed) & $2.66 \pm 0.42$ & $-0.13 \pm 0.29$ \\
\hline
\end{tabular}

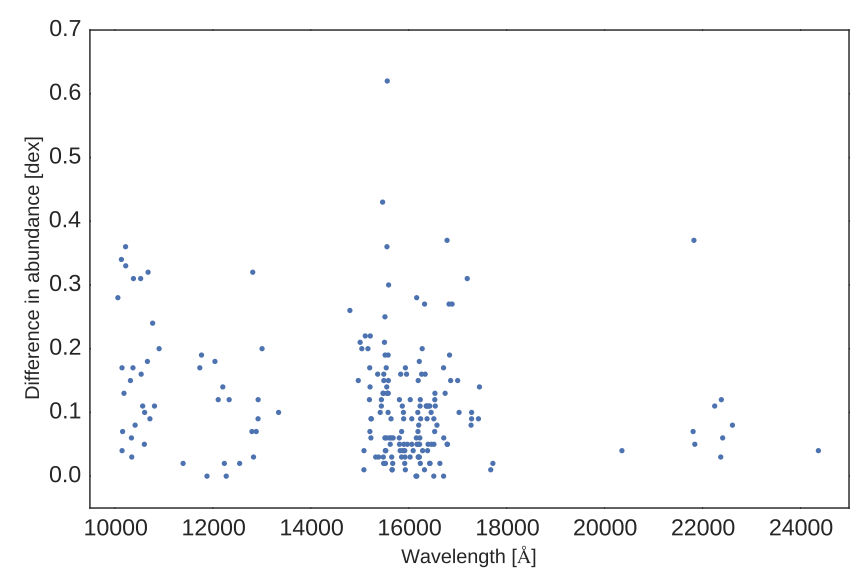

Fig. 7. Difference in abundance for lines in HD 20010 that have at least two measurements of EW. The difference is calculated between the highest measured EW and the lowest. For the line list used for HD 20010 we used the mean of all measurements available.

atmospheric parameters, we made a $3 \sigma$ outlier removal in the abundance iteratively until there were no more outliers present. Since we could only measure 5 Fe II lines, for comparison we also decided to derive parameters using the same method, but we fixed the surface gravity to the reference value. The resulting atmospheric parameters and iron abundances are presented in Table 4. The effective temperature, surface gravity, and metallicity agree within the errors with the literature values. Similar parameters are obtained by fixing $\log g$ to the average literature value or by leaving it free.

The errors on the atmospheric parameters for HD 20010 are much higher than what is achievable with other measurements in the literature, as presented above in Table 3. In order to explain these errors, we calculated the abundances for all lines which have at least two measurements of the EW. We then calculated
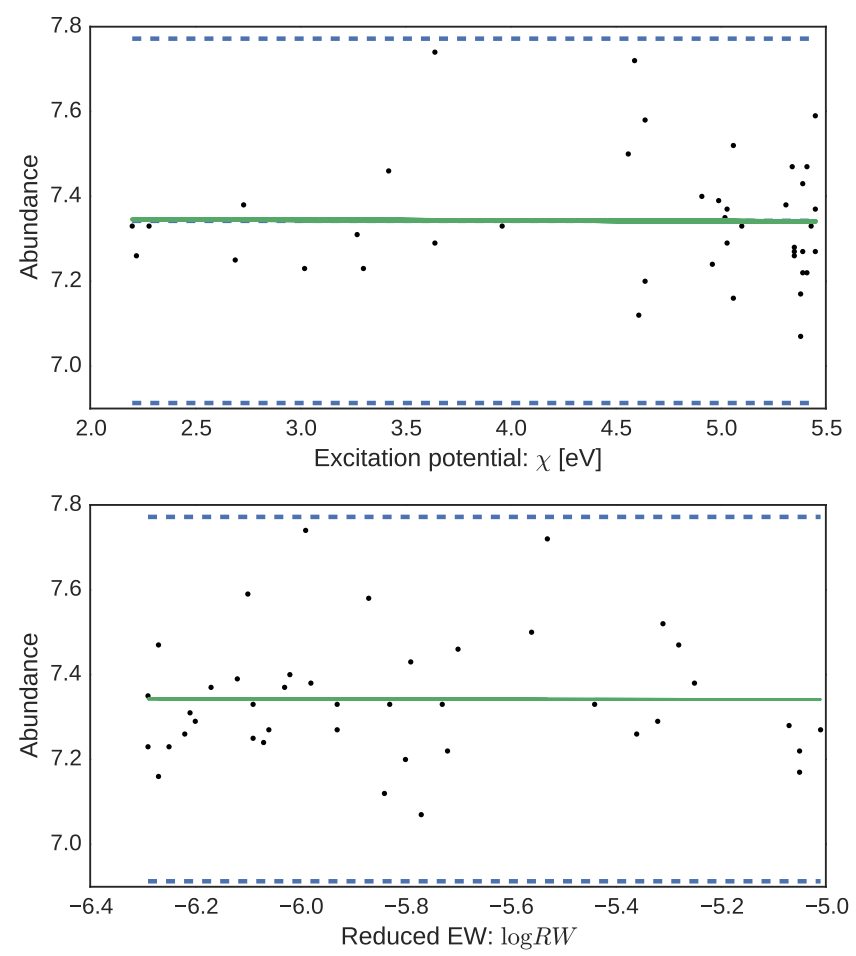

Fig. 8. Top plot: Fe I abundances for all lines are shown as a function of EP. Bottom plot: Fe I abundances, but against the reduced EW. The high dispersion in the abundances leads to high error bars on the derived atmospheric parameters. The green lines are the slopes, and the dashed lines are the mean (under the green line), and the $3 \sigma$ standard deviation.

the abundances for the highest measured EW and the lowest. The differences in abundances are presented in Fig. 7. The very large differences (more than $0.1 \mathrm{dex}$ ) translate to the high errors in the parameters.

The source of the large errors on the parameters can be seen more clearly where abundances are compared to excitation potential or abundances versus reduced EW. Here the dispersion on the abundances can be seen clearly, as shown in Fig. 8 .

This test strongly suggest that errors in the EWs, likely due to the poor quality of this spectrum, are responsible for the relatively large error bars in the derived stellar parameters. Systematic errors (e.g. due to a possible non-optimal reduction of the spectrum) may be the reason for these large error bars. As 
the CRIRES-POP team continue their great efforts in reducing the optimal spectra, it will be interesting to re-visit this star once the entire spectrum has been fully reduced.

\subsubsection{Surface gravity}

Although we have derived a consistent value for the surface gravity for HD 20010, given the small number of Fe II lines in the analysis, we find this value to be of low precision and it should be considered with caution. However, we emphasize that from our experience in using this method (the ionization balance) in the optical, the other atmospheric parameters $\left(T_{\text {eff }}\right.$, and $[\mathrm{Fe} / \mathrm{H}]$ ) have a low interdependency with the surface gravity. This has been shown by Torres et al. (2012) and more recently by Mortier et al. (2014). Furthermore, with the upcoming results from the Gaia mission we will get precise surface gravity for a large number of stars and thus the best option would be to fix this parameter if necessary.

\section{Conclusion}

In this work, we present a new iron line list for the NIR. The quality of the line list plays a key role in deriving atmospheric stellar parameters. Although the line list was compiled from a solar spectrum and calibrated for the same, we tested it extensively for the slightly hotter star HD 20010. The first results with this line list are promising. We also show that for a spectrum that contains telluric lines, the best results appear when removing lines with an EW lower than $5 \mathrm{~m} \AA$. In the future, the development of new high resolution NIR spectrographs will allow us to obtain more high quality spectra of stars in the whole FGK spectral range, thus allowing us to better test and refine this line list.

Furthermore, it will be interesting to explore the use of this line list to derive parameters for M-dwarf stars using high resolution and high signal-to-noise NIR spectra. M-dwarf stars are especially interesting targets for an exoplanetary viewpoint, since they are prone to forming low-mass exoplanets (Bonfils et al. 2013). Hence, a precise analysis of the host star's atmospheric parameters may greatly improve our characterization of the possible exoplanets orbiting these low-mass stars.

Lastly, with the upcoming NIR spectrographs discussed above, this work and future continuation will help the community to derive atmospheric stellar parameters.

Acknowledgements. This work was supported by Fundação para a Ciência e a Tecnologia (FCT) through the research grants UID/FIS/04434/2013 and PTDC/FIS-AST/1526/2014. N.C.S., and S.G.S. acknowledge the support from FCT through Investigador FCT contracts of reference IF/00169/2012, and IF/00028/2014, respectively, and POPH/FSE (EC) by FEDER funding through the program "Programa Operacional de Factores de Competitividade - COMPETE". E.D.M. and B.J.A. acknowledge the support from FCT in form of the fellowship SFRH/BPD/76606/2011 and SFRH/BPD/87776/2012, respectively. This work also benefited from the collaboration of a cooperation project FCT/CAPES - 2014/2015 (FCT Proc 4.4.1.00 CAPES). This research has made use of the SIMBAD database operated at CDS, Strasbourg (France). This work has made use of the VALD database, operated at Uppsala University, the Institute of Astronomy RAS in Moscow, and the University of Vienna. We thank the anonymous referee for useful comments and suggestions. Finally, a special thanks to Dr. Thomas Lebzelter from the CRIRES-POP team for helping with various questions on the spectra of HD 20010.

\section{References}

Ammler-von Eiff, M., Santos, N. C., Sousa, S. G., et al. 2009, A\&A, 507, 523 Artigau, É., Kouach, D., Donati, J.-F., et al. 2014, in SPIE Conf. Ser., 9147, 15
Balachandran, S. 1990, ApJ, 354, 310

Baraffe, I., Homeier, D., Allard, F., \& Chabrier, G. 2015, A\&A, 577, A42

Bensby, T., Feltzing, S., \& Oey, M. S. 2014, A\&A, 562, A71

Bertaux, J. L., Lallement, R., Ferron, S., Boonne, C., \& Bodichon, R. 2014, A\&A, 564, A46

Blackwell, D. E., \& Shallis, M. J. 1977, MNRAS, 180, 177

Blanco-Cuaresma, S., Soubiran, C., Heiter, U., \& Jofré, P. 2014, iSpec: Stellar atmospheric parameters and chemical abundances (Astrophysics Source Code Library)

Bonfils, X., Delfosse, X., Udry, S., et al. 2013, A\&A, 549, A109

Boyajian, T. S., von Braun, K., van Belle, G., et al. 2012, ApJ, 757, 112

Casagrande, L., Ramírez, I., Meléndez, J., Bessell, M., \& Asplund, M. 2010, A\&A, 512, A54

Cayrel, R. 1988, in The Impact of Very High S/N Spectroscopy on Stellar Physics, eds. G. Cayrel de Strobel, \& M. Spite, IAU Symp., 132, 345

Delfosse, X., Donati, J.-F., Kouach, D., et al. 2013, in SF2A-2013: Proc. Annual meeting of the French Society of Astronomy and Astrophysics, eds. L. Cambresy, F. Martins, E. Nuss, \& A. Palacios, 497

Dotter, A., Chaboyer, B., Jevremović, D., et al. 2008, ApJS, 178, 89

Favata, F., Micela, G., \& Sciortino, S. 1997, A\&A, 323, 809

Follert, R., Dorn, R. J., Oliva, E., et al. 2014, in SPIE Conf. Ser. 9147, 19

Girardi, L., Bressan, A., Bertelli, G., \& Chiosi, C. 2000, A\&AS, 141, 371

Gonzalez, G., \& Laws, C. 2000, AJ, 119, 390

Gonzalez, G., \& Vanture, A. D. 1998, A\&A, 339, L29

Gonzalez, G., Carlson, M. K., \& Tobin, R. W. 2010, MNRAS, 403, 1368

Gray, D. F. 2005, The Observation and Analysis of Stellar Photospheres, 3rd edn.

Gustafsson, B., Edvardsson, B., Eriksson, K., et al. 2008, A\&A, 486, 951

Hinkle, K. H., Wallace, L., \& Livingston, W. 1995, in Laboratory and Astronomical High Resolution Spectra, eds. A. J. Sauval, R. Blomme, \& N. Grevesse, ASP Conf. Ser., 81, 66

Kjeldsen, H., \& Bedding, T. R. 1995, A\&A, 293, 87

Kotani, T., Tamura, M., Suto, H., et al. 2014, in SPIE Conf. Ser., 9147, 14

Kupka, F. G., Ryabchikova, T. A., Piskunov, N. E., Stempels, H. C., \& Weiss, W. W. 2000, Balt. Astron., 9, 590

Kurucz, R. 1993, ATLAS9 Stellar Atmosphere Programs and $2 \mathrm{~km} \mathrm{~s}^{-1}$ grid. Kurucz CD-ROM No. 13 (Cambridge, Mass.: Smithsonian Astrophysical Observatory), 13

Lebzelter, T., Seifahrt, A., Uttenthaler, S., et al. 2012, A\&A, 539, A109

Meléndez, J., \& Barbuy, B. 1999, ApJS, 124, 527

Mortier, A., Santos, N. C., Sousa, S., et al. 2013, A\&A, 551, A112

Mortier, A., Sousa, S. G., Adibekyan, V. Z., Brandão, I. M., \& Santos, N. C. 2014, A\&A, 572, A95

Mucciarelli, A., Pancino, E., Lovisi, L., Ferraro, F. R., \& Lapenna, E. 2013, ApJ, 766, 78

Önehag, A., Heiter, U., Gustafsson, B., et al. 2012, A\&A, 542, A33

Origlia, L., Oliva, E., Maiolino, R., et al. 2013, A\&A, 560, A46

Origlia, L., Oliva, E., Baffa, C., et al. 2014, in SPIE Conf. Ser., 9147, 1

Piskunov, N. E., Kupka, F., Ryabchikova, T. A., Weiss, W. W., \& Jeffery, C. S. 1995, A\&AS, 112, 525

Press, W. H., Teukolsky, S. A., Vetterling, W. T., \& Flannery, B. P. 1992, Numerical Recipes in C, 2nd edn.): the Art of Scientific Computing (New York: Cambridge University Press)

Quirrenbach, A., Amado, P. J., Caballero, J. A., et al. 2014, in SPIE Conf. Ser., 1

Ramírez, I., \& Meléndez, J. 2005, ApJ, 626, 446

Ramírez, I., Fish, J. R., Lambert, D. L., \& Allende Prieto, C. 2012, ApJ, 756, 46 Recio-Blanco, A., Bijaoui, A., \& de Laverny, P. 2006, MNRAS, 370, 141

Rhodin, H. 2015, Determining the effective temperature of cool stars from nearinfrared spectra, student Paper

Santos, N. C., Israelian, G., \& Mayor, M. 2004, A\&A, 415, 1153

Santos, N. C., Sousa, S. G., Mortier, A., et al. 2013, A\&A, 556, A150

Shetrone, M., Bizyaev, D., Lawler, J., et al. 2015, ApJS, submitted [arXiv: 1502.04080 ]

Smith, V. V., Cunha, K., Shetrone, M. D., et al. 2013, ApJ, 765, 16

Sneden, C. A. 1973, Ph.D. Thesis, The University of Texas at Austin

Sousa, S. G. 2014, ArXiv e-prints [arXiv: 1407. 5817]

Sousa, S. G., Santos, N. C., Israelian, G., Mayor, M., \& Monteiro, M. J. P. F. G. 2007, A\&A, 469, 783

Sousa, S. G., Santos, N. C., Mayor, M., et al. 2008, A\&A, 487, 373

Sousa, S. G., Santos, N. C., Adibekyan, V., Delgado-Mena, E., \& Israelian, G. 2015, A\&A, 577, A67

Torres, G., Winn, J. N., \& Holman, M. J. 2008, ApJ, 677, 1324

Torres, G., Fischer, D. A., Sozzetti, A., et al. 2012, ApJ, 757, 161

Tsantaki, M., Sousa, S. G., Adibekyan, V. Z., et al. 2013, A\&A, 555, A150

Valenti, J. A., \& Fischer, D. A. 2005, ApJS, 159, 141 
D. T. Andreasen et al.: Near-infrared spectroscopy of the Sun and HD 20010

\section{Appendix A: Iron abundances before recalibrating $\log g \boldsymbol{f}$}

It is clear from Fig. A.1 that most of the lines taken from VALD3 have bad $\log g f$ values. This reinforces our need to use differential analysis also in the NIR.

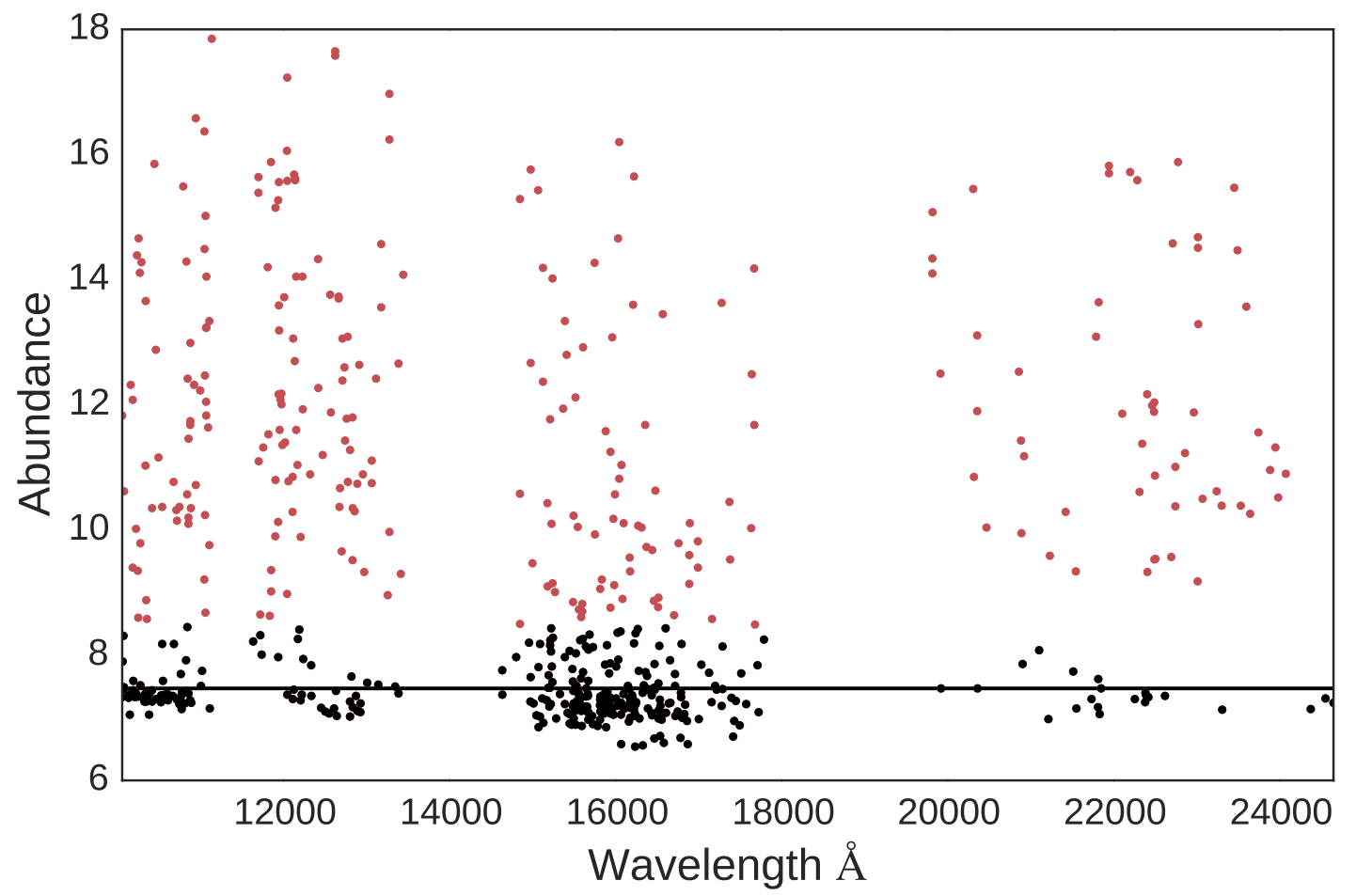

Fig. A.1. Abundances of all Fe I lines before recalibration of the $\log g f$ values as a function of the wavelength. All red points deviate more than 1 dex from the expected solar value of 7.47 (horizontal line) and are therefore discarded from the line list. 